\title{
Software Tool for Testing the Packet Analyzer of Network Attack Detection Systems
}

\author{
Alexander Branitskiy \\ Saint-Petersburg Institute for Informatics and \\ Automation of the Russian Academy of Sciences \\ 14-th Liniya, 39, St.Petersburg, 199178, Russia \\ St. Petersburg National Research University of \\ Information Technologies, Mechanics and Optics, 49, \\ Kronverkskiy prospekt, St.Petersburg, Russia \\ branitskiy@comsec.spb.ru
}

\author{
Igor Kotenko \\ Saint-Petersburg Institute for Informatics and \\ Automation of the Russian Academy of Sciences \\ 14-th Liniya, 39, St.Petersburg, 199178, Russia \\ St. Petersburg National Research University of \\ Information Technologies, Mechanics and Optics, 49, \\ Kronverkskiy prospekt, St.Petersburg, Russia \\ ivkote@comsec.spb.ru
}

\begin{abstract}
$^{\mathbf{1}}$
The paper is devoted to a model, technique and software tool for testing network attack detection systems (ADSs) from the point of view of the correct functioning of their internal packet analyzer. A client-server architecture of the software tool for generating the low-level network attacks is described. The paper outlines the experimental results of testing several ADSs by using the implemented software tool. The results of experiments, aimed at testing the ADSs on the ability to detect evasion and insertion attacks, are presented. We analyze the reaction of ADSs in response to various network packets subjected to various transformations at the levels both of IP and TCP protocols. Recommendations aimed at detecting and preventing such situations in computer networks are proposed.
\end{abstract}

\section{CCS CONCEPTS}

- Security and privacy $\rightarrow$ Intrusion/anomaly detection and malware mitigation

\section{KEYWORDS}

TCP/IP protocols, network attacks, packet analyzer, attack detection systems

\section{ACM Reference format:}

A. Branitskiy and I. Kotenko, 2018. Software Tool for Testing the Packet Analyzer of Network Attack Detection Systems. In SIN '18: International Conference On Security Of Information and Networks, 8 pages. DOI: $10.1145 / 3264437.3264488$

\footnotetext{
${ }^{1}$ Permission to make digital or hard copies of all or part of this work for personal or classroom use is granted without fee provided that copies are not made or distributed for profit or commercial advantage and that copies bear this notice and the full citation on the first page. Copyrights for components of this work owned by others than ACM must be honored. Abstracting with credit is permitted. To copy otherwise, or republish, to post on servers or to redistribute to lists, requires prior specific permission and/or a fee. Request permissions from Permissions@acm.org.

SIN '18, September 10-12, 2018, Cardiff, United Kingdom

(C) 2018 Association for Computing Machinery.

ACM ISBN 978-1-4503-6608-3/18/09...\$15.00

https://doi.org/10.1145/3264437.3264488
}

\section{INTRODUCTION}

In the age of digital technology one of the key problems in modern society is the protection of information. This leads to the creation of certain systems and tools that should have in their presence at the network level some mechanisms for protecting and countering hacker threats. Currently it is considered important to provide the highest level of security in corporate networks of any organization that stores critical data. The success and competitiveness of such an organization largely depends on the usage of effective tools that satisfy the conditions above $[1,2]$.

One of the most well-known and well-proven tools for solving this problem are the ADSs. By the way of data collecting the ADSs are host-based and network. The host-based ADSs are oriented to the monitoring of operating system (OS) processes and devices connected to a certain host. The network ADSs can be defined as software or hardware devices designed to detect unauthorized access attempts and security violations in the context of network interaction between hosts.

In this paper we put more emphasis on the analysis of network ADSs from the point of view of the correct functioning of their internal packet analyzer.

The novelty of the research is as follows. First, we propose an architecture of a software tool for generating low-level network attacks, which, unlike other analogues, is client-server, supports multiple connections from heterogeneous thin clients with the possibility of online transformation of network packets, and provides a mechanism of proxying for TCP-sessions (hiding the IP-address of the source of attacks). Secondly, detailed testing of network ADSs at four levels of the OSI model was performed, and the results of corresponding experiments for three ADSs listening for traffic directed to two network services on two OSs were obtained.

The rest of the paper is organized as follows. The section 2 is related work. The section 3 represents the model of checking the packet analyzer. The section 4 reveals the proposed tool aimed at testing the network ADSs. In the section 5 we present the results of the carried out experiments. The section 6 is the discussion of the results obtained. The section 7 contains the conclusion and further possible directions. 


\section{RELATED WORK}

In Table 1 we have presented some papers which are directed on investigation of different network ADSs.

Table 1: Related Work

\begin{tabular}{|c|c|c|}
\hline $\begin{array}{l}\text { Ref., } \\
\text { year }\end{array}$ & ADSs & Brief content \\
\hline $\begin{array}{l}{[3],} \\
2011\end{array}$ & $\begin{array}{l}\text { Snort, } \\
\text { Suricata }\end{array}$ & $\begin{array}{l}\text { The investigation is aimed at comparison of ADSs } \\
\text { on the consumption of system resources (the } \\
\text { dependence of the processor load on the } \\
\text { bandwidth of the network channel, the time of } \\
\text { reading pcap-files in offline mode, the usage of } \\
\text { several CPU cores, the dependence of the number } \\
\text { of discarded packets on the availability of the } \\
\text { CPU), the number of false positives and the } \\
\text { number of correctly detected attacks. Suricata is } \\
\text { characterized greater accuracy in detecting attacks, } \\
\text { more uniform usage and distribution of tasks } \\
\text { (threads) between processor cores, faster } \\
\text { processing of traffic while increasing the number } \\
\text { of involved cores, and slower processing of the } \\
\text { pcap file. Snort demonstrates less stable load } \\
\text { balancing between cores and a high speed of } \\
\text { reading the network dumps. }\end{array}$ \\
\hline $\begin{array}{l}{[4],} \\
2003\end{array}$ & $\begin{array}{l}\text { Dragon, } \\
\text { Firestorm, } \\
\text { Snort, } \\
\text { Prelude }\end{array}$ & $\begin{array}{l}\text { The paper includes comparison of several ADSs } \\
\text { by the criterion of detection of network attacks } \\
\text { using the example of DARPA } 1999 \text { network } \\
\text { dumps. During the tests the best results was shown } \\
\text { by a commercial ADS Dragon. }\end{array}$ \\
\hline $\begin{array}{l}{[5],} \\
2013\end{array}$ & $\begin{array}{l}\text { Snort, } \\
\text { Suricata }\end{array}$ & $\begin{array}{l}\text { The paper is devoted to a comparative analysis of } \\
\text { two ADSs. The experiments were conducted to } \\
\text { determine the regularities of the amount of } \\
\text { consumed resources (CPU load, the amount of } \\
\text { RAM consumed) and the processing speed of } \\
\text { packets, depending on the number of cores used in } \\
\text { the system and in the ADS settings. It was noted } \\
\text { that Suricata has the best performance. }\end{array}$ \\
\hline $\begin{array}{l}{[6],} \\
2015\end{array}$ & $\begin{array}{l}\text { Snort, } \\
\text { Suricata }\end{array}$ & $\begin{array}{l}\text { The paper considers several experiments aimed at } \\
\text { testing the ADSs. These tests are the following: } \\
\text { determining the speed of processing the pcap-file, } \\
\text { generating attacks to determine the accuracy of } \\
\text { their detection, determining the consumption of } \\
\text { system resources of each of the ADS, } \\
\text { determining the accuracy of detecting attacks with } \\
\text { background "white" traffic, using a set of running } \\
\text { ADSs. Snort shows more economical usage of } \\
\text { system resources. Suricata demonstrates greater } \\
\text { accuracy in detecting attacks and less time spent } \\
\text { on reading a pcap-file. }\end{array}$ \\
\hline $\begin{array}{l}{[7],} \\
2011\end{array}$ & $\begin{array}{l}\text { Snort, } \\
\text { Suricata }\end{array}$ & $\begin{array}{l}\text { The paper compares two ADSs on the basis of } \\
\text { their usage in the following experiments: } \\
\text { measuring the performance of ADSs on a real } \\
\text { traffic and on a static pcap file (packet reading } \\
\text { speed), taking into account the system resources } \\
\text { spent on processing of packets (CPU and RAM } \\
\text { resources usage), determining the correct detection } \\
\text { of attacks. According to the obtained results } \\
\text { Suricata shows a higher speed of traffic processing } \\
\text { due to the multithreaded architecture and, as a } \\
\text { consequence, requires more system resources } \\
\text { (memory and CPU time) during its operating. The } \\
\text { bandwidth of Snort is limited to } 200-300 \mathrm{Mb} / \mathrm{sec} \text {, }\end{array}$ \\
\hline
\end{tabular}

\begin{tabular}{|l|l|l|}
\hline $\begin{array}{l}\text { Ref., } \\
\text { year }\end{array}$ & ADSs & \multicolumn{1}{|c|}{ Brief content } \\
\hline [8], & Snort, Bro & $\begin{array}{l}\text { and the procedure for using the multiple Snort } \\
\text { instances is losing one compared to running one } \\
\text { copy of Suricata. }\end{array}$ \\
\hline 2008 & $\begin{array}{l}\text { The paper is a review of two ADSs. The internal } \\
\text { architecture of each of the systems is described, } \\
\text { the rules for detecting attacks are provided. } \\
\text { Several distinctive features were revealed when } \\
\text { analyzing the network traffic with help of ADSs. It } \\
\text { is noted that Snort operates on a packet level and } \\
\text { analyzes both individual raw IP fragments and the } \\
\text { reconstructed network stream, while Bro analyzers } \\
\text { are started only after the complete } \\
\text { defragmentation of packets. }\end{array}$ \\
\hline [9], \\
2011 & $\begin{array}{l}\text { Snort, } \\
\text { Suricata, } \\
\text { Bro }\end{array}$ & $\begin{array}{l}\text { The purpose of the investigation is to compare the } \\
\text { performance indicators of the considered ADSs. } \\
\text { Testsere made to determine the processing time } \\
\text { generated warnings during its reading, and to } \\
\text { detect attacks in traffic generated using the } \\
\text { Metasploit tool. }\end{array}$ \\
\hline
\end{tabular}

This paper is aimed at a thorough investigation of the packet analyzer of each of ADSs (Snort [10], Suricata [11] and Bro [12]). The contribution of the paper is a formal description of a model of checking the packet analyzer, representation of the developed software tool, experimental verification of each ADSs and recommendations for the normal operation of the packet analyzer.

\section{MODEL OF CHECKING THE PACKET ANALYZER}

To describe the process which determines the checking of the packet analyzer we introduce the following model:

$$
C P A=<S, V, P, A^{(O S)}, A^{(A D S)}, C>,
$$

where $S=\left\{s_{i}\right\}^{N}{ }_{i=1} \subset B^{+}$is a set of attack scenarios, $B=\{0, \ldots, \mathrm{M}\}$ is a set of byte symbols $(M=255), B^{+}=\{0,1, \ldots, \mathrm{M}, 00,01, \ldots, 0 \mathrm{M}, \ldots\}$ is a set of byte sequences (all possible packets), $V \in\left\{0, \ldots,(\mathrm{M}+1)^{4}-1\right\}$ is a victim host (its IP-address), $P \subset\left\{0, \ldots,(\mathrm{M}+1)^{2}-1\right\}$ is a list of opened ports of the host $V$ and network applications associated with them, $A^{(O S)}, A^{(A D S)}: 2^{B+} \rightarrow B^{+}$ is a mappings which transform several input packets into a new output byte sequence with help of the operating system network stack and ADS network analyzer accordingly, $C: B^{+} \times B^{+} \rightarrow\{0,1\}$ is a checking procedure which can be expressed as a predicate of equality of the first and second arguments, e.g. as follows via Iverson notation: $C(x, y)=[x=y]$.

According to this model an attacker tries to form such sequence of network packets which, when assembled by the OS kernel on the target host, will differ from the data analyzed by the ADS. In accordance with the paper [13] such actions can lead to (1) evasion attacks [14] and (2) insertion attacks [14].

In the first case the attacker's goal is to create such packets, some of which are ignored by the network analyzer of ADS, but processed by the OS network stack (Fig. 1). A typical example of such an attack is the sending of payload data in the first packet of TCP-session. If the OS kernel is configured to process the data contained in the SYN-packet, and the packet analyzer of ADS does not take into account this feature, then this can lead to a serious gap in the protected host, namely, a network attack skip. 


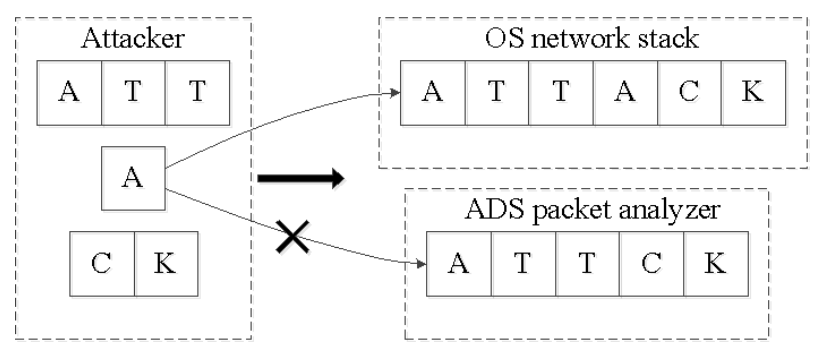

Figure 1: Illustration of evasion attack.

The second case is characterized by the fact that the packet analyzer of ADS processes those packets that are not taken into account by the OS network stack (Fig. 2). An example of such an attack is sending packets with time pauses between them. If the timeout allocated to the assembly of the network stream using the ADS packet analyzer is $T^{(A D S)}$, and with the OS network stack $T^{(O S)}$, and $T^{(A D S)}>T^{(O S)}$ is satisfied, then it becomes possible to insert a dummy packet into the ADS analysis process.

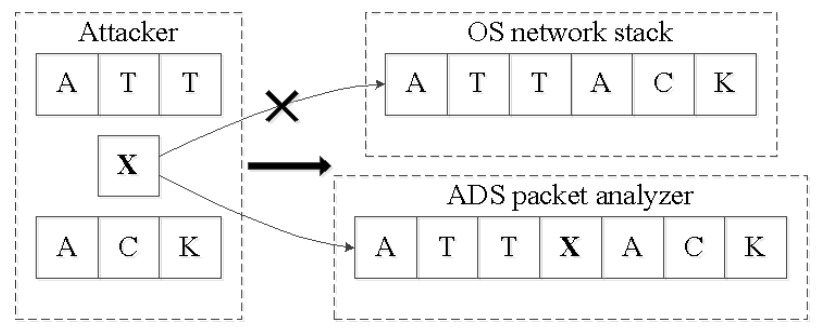

Figure 2: Illustration of insertion attack.

\section{TOOL FOR TESTING THE PACKET ANALYZER}

For testing the network ADSs we have developed a special software tool which (1) is designed for transparent proxying of asynchronously incoming client requests inside the TCP connections, (2) supports the generation of test sequences of lowlevel network attacks, (3) allows to perform automated execution of a number of network attacks on the protected host in order to bypass one of the ADSs launched on it . This tool is called an asynchronous transparent proxy-server of TCP-sessions, which general architecture is represented in Fig. 3. It consists of three components. The first component of (reader) is a sniffer with an extended set of functions which, in addition to reading and parsing of network packets from the interface and pcap-file, is capable to "replay" the network traffic into the interface with a given sending rate, change source and destination IP-addresses with checksums and filter certain packets according to the specified BPF. The second component of (sender) is designed to fill the fields of an IP-packet with any type of protocol (TCP, UDP, ICMP or manually generated by the user) and to send this packet through a raw socket (among open source tools with similar functionality we can call sendip [15] and hping3 [16]). This component can be launched both in usual mode and in the daemon mode. In the first case the package data are formed from the command-line arguments, in the second case the control commands are read from the named UNIX pipes. The role of the third component (processor) is to perform the replacement of some packets and provide the interaction between the first two components.

Fig. 3 shows $\mathrm{N}$ thin clients which connect to the proxy-server through the standard TCP sockets. The proxy-server which listens to them can be launched both locally and on a separate machine. Client ports can be selected both (1) automatically with help of built-in OS resources by calling the function "connect" with the arguments of the client socket descriptor passed to it and the proxy-server address along with the port, and (2) manually using a sequence of function calls "bind" and "connect".

On the side of the proxy-server three processes are started, corresponding to the above components: reader, sender and pkt processor. The processor component handles packets coming from client applications and searches a given signature sequence in each of these packets. If the signature is a substring of the content of the captured packet, then this packet is subjected to certain transformations, which depend on the type of attack scenario chosen by the attacker, e.g. hidden IP-fragmentation, sending packets with delays, setting inconsistent flags in the TCP-packet header, changing the sequence of packets, etc.). When the new ith client (client_addr_i, client_port_i) is connected to the port proxy_server_port of the proxy server, the considered component creates the i-th entry in the proxy-table. In this table there are data of all incoming connections which are registered on the port proxy_server_port. Simultaneously with adding this record into the proxy table, the port raw_proxy_port_ $i$ is searched for and reserved, and its correspondence to the $\mathrm{i}$-th client socket is assigned. Through the port raw_proxy_port $\mathrm{i}$ the network interaction is performed between the proxy-server and the host being attacked (victim_addr, victim_port) and emulated using a raw socket. As soon as packets arrive from the i-th client (or from the attacked host to the port raw_proxy_port $i$ of the proxyserver), these packets are automatically redirected according to the i-th entry in the proxy-table. Thus for the client data exchange with the host being attacked occurs transparently through some intermediary (proxy-server) that provides some packet transformation services.

Unlike the popular such tools as fragrouter [17] and fragroute [18], which are also designed to perform similar network attacks, the developed software uses a client-server architecture and allows to generate a much greater variety of attack scenarios (comparison of these tools is presented in Table 2).

Table 2: Comparison of network tools

\begin{tabular}{|c|c|c|c|}
\hline \multirow{2}{*}{ Characteristic } & \multicolumn{3}{|c|}{ Compared tools } \\
\cline { 2 - 4 } & $\begin{array}{c}\text { fragroute/ } \\
\text { fragrouter }\end{array}$ & $\begin{array}{c}\text { hping3/ } \\
\text { sendip }\end{array}$ & $\begin{array}{c}\text { Developed } \\
\text { tool }\end{array}$ \\
\hline $\begin{array}{c}\text { Presence of client-server } \\
\text { architecture }\end{array}$ & - & - & + \\
\hline $\begin{array}{c}\text { Support of proxying the } \\
\text { TCP-sessions }\end{array}$ & - & - & + \\
\hline $\begin{array}{c}\text { Covered levels of the OSI } \\
\text { model }\end{array}$ & 3,4 & 3,4 & $2,3,4,7$ \\
\hline $\begin{array}{c}\text { Transformation of packets } \\
\text { within the TCP-sessions }\end{array}$ & - & - & + \\
\hline $\begin{array}{c}\text { Presence of scanning } \\
\text { function }\end{array}$ & - & + & - \\
\hline
\end{tabular}




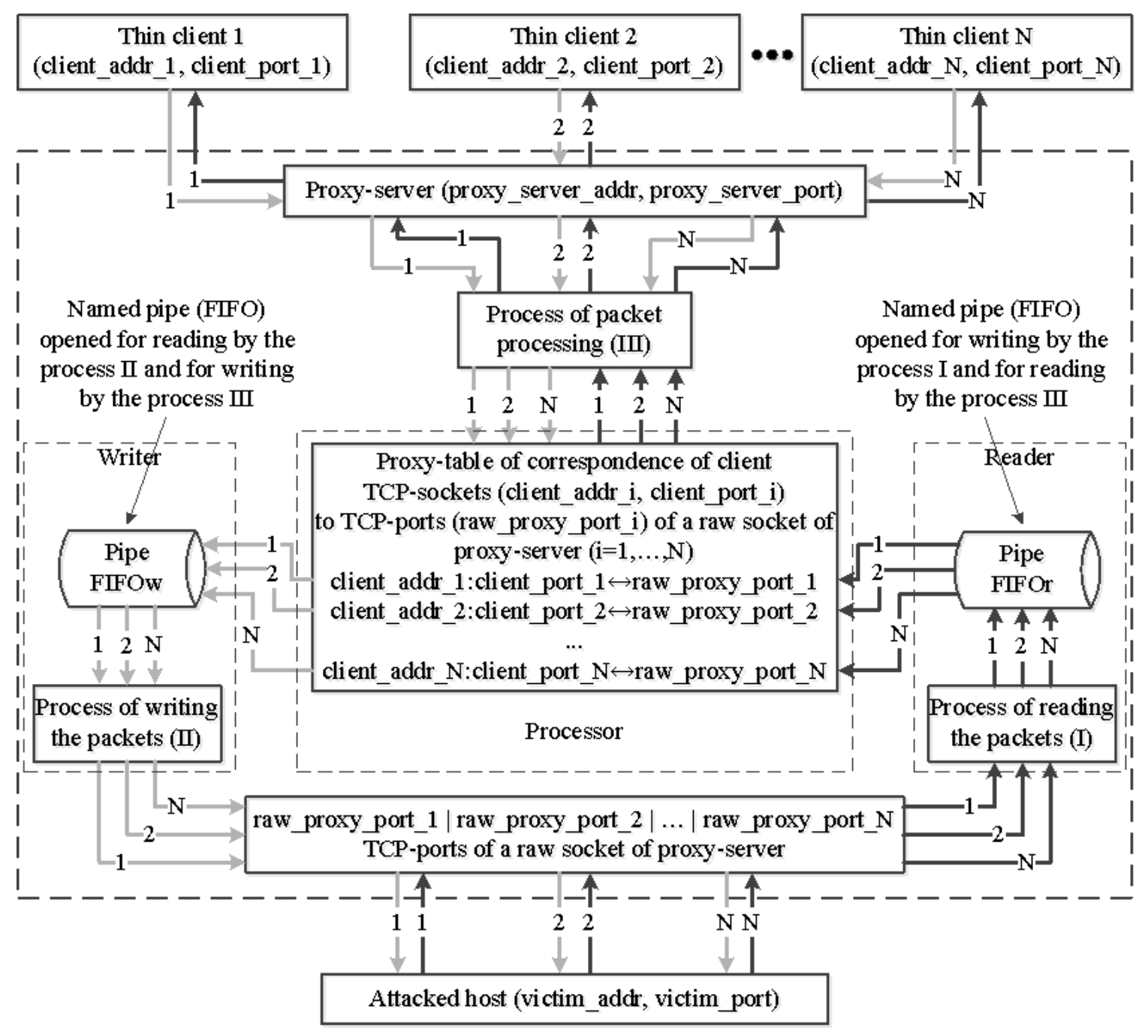

Figure 3: Asynchronous transparent proxy-server of TCP-sessions.

It should also be noted that by setting the rules of the standard Linux built-in firewall on a machine with a proxy-server installed, outgoing packet with RST-flag send is forbidden. Thereby the OS network stack ignores incoming packets which are addressed to the closed port of the raw socket (it is closed because it is not registered in the OS as being used). Therefore the remote server "thinks" that it communicates with the real application, although in fact some conversion of packets forwarded from thin clients is performed.

The algorithm of functioning the asynchronous transparent proxy-server of TCP-sessions is represented as follows. The first component (reader) sends packets from the attacked host to the FIFOr queue in the post-processed string representation. The second component (writer) creates a network channel in which the data are transmitted bypassing the OS network stack at the raw socket level. It should also be noted that by setting the rules of the standard built-in Linux firewall on a machine with a proxy server installed, it was forbidden to send outgoing RST-packets so that the OS network stack ignored incoming packets to the closed port of the raw socket. It is closed because it is not registered in the OS as being used. Thus, such a reception creates the appearance of the remote server that it communicates with the real application, although in fact some conversion of packets forwarded from thin clients to the remote server is carried out. A rule which blocks the sending of such packets at the kernel level with the outgoing port raw proxy_port $\mathrm{i}$ is specified using the following command: iptables -A OUTPUT -p tcp --tcp-flags RST RST -j DROP --sport raw_proxy_port_i. A command that cancels changes made by the previous rule, is iptables -D OUTPUT -p tcp --tcp-flags RST RST -j DROP --sport raw_proxy_port_i. The third component (processor) launches a TCP-socket with listening on incoming connections on the port 9000 and transparently redirects requests from thin clients connected to it to the remote attacked server. To this end, it writes data to the string representation of packets into the FIFOw queue of the second component, which captures this record, generates a real packet, and sends it to the network interface to the remote server side.

Before sending some packets directly to the server, they are modified according to one of the rules which define the attack scenarios described in the section 5 . 
When a new incoming thin client connection is registered, no new thread is created at the input of the asynchronous transparent proxy-server of TCP-sessions. Instead of this the descriptor of the corresponding client socket is put in the EPOLL-queue to monitor its state and check the data ready to read from this descriptor. Since all the functioning of the proxy-server is contained in one loop, the computing resources are saved by reducing the costs associated with creating a new thread for processing client connections.

The peculiarities of the developed software are the following:

- partial emulation of TCP/IP interaction at the level of raw sockets:

- automatic calculation of checksums on IP- and TCP-levels;

- automatic calculation of the values of the next sequence numbers and acknowledgment numbers;

- the correct establishment and termination of the TCP-session;

- $\quad$ automatic calculation of IP-identifiers;

- automatic calculation of offsets for IP-fragments;

- transparent proxying of TCP/IP-connections;

- support for multiple connections from different clients;

- asynchronous processing of requests from thin clients;

- single-threaded functioning;

- modular construction (all low-level parts for operating with the network are written in $\mathrm{C}$, the component of their interaction is implemented through a script in Perl);

- generation of test sequences of low-level network attacks, mainly aimed at fragmentation of packets, setting the various header field values from the lowest level to the highest level.

\section{EXPERIMENTS}

Fig. 4 presents the software stand used for conducting the experiments. In the virtual environment KVM (Kernel-based Virtual Machine) three machines were deployed: the first host is an attacker (10.0.1.100), the second (10.0.1.101) and the third (10.0.1.102) hosts provide access to some of the network services running on them (HTTP and FTP) and include the network ADSs installed on each of these hosts.

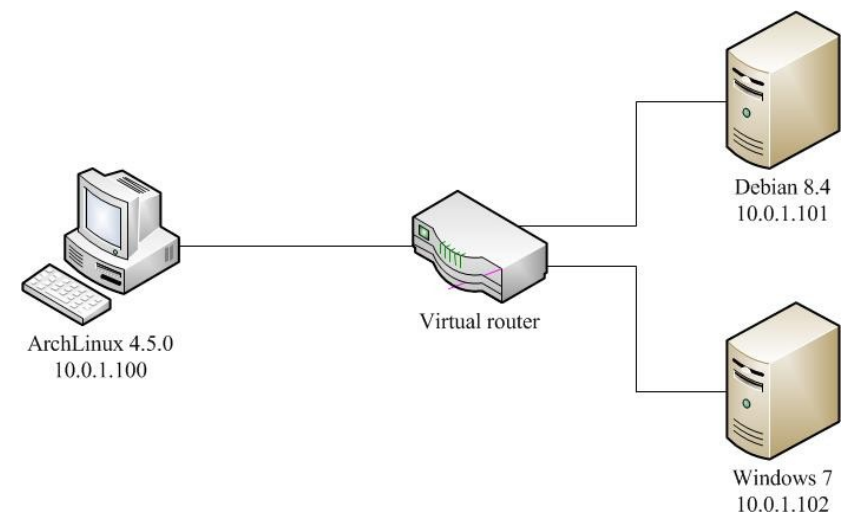

Figure 4: Software stand for testing the network ADSs.
As services we have used HTTP (Web) and FTP. From the side of the protected machines we have installed the server applications apache2 2.4.20 and vsftpd 3.0.2-17 for Linux 3.16.04, nginx 1.10.0 and MS FTP Service for Windows 7. From the side of attacking machine we have utilized thin clients - the developed and simplified interactive analog of wget/curl and the program ftp running in passive mode.

The test substrings which have been defined in the signature rules of ADSs are HTTP- and FTP-requests (GET and RETR) for loading a specific file located on the server 10.0.1.101 or 10.0.1.102. If content of some captured network packet contains this request then such packet is transformed. As the basis for these signatures we have used the standard Snort rule with the identifier 1762 which is designed for detecting the well-known vulnerability on the Web-server (execution of arbitrary code on the remote machine with superuser rights throughout running the CGI-script). Note that attacks which are aimed at a primitive circumvention of the signature rule are not considered, some of which include deliberate insertion of additional insignificant spaces between the command and its parameters, changing the register in commands and their arguments, or replacing the standard path to the file with some other equivalent absolute or relative path to the same file.

Fig. 5 shows test Snort signature rules for finding substrings "GET/secret.html" and "RETR confidential_info.txt" in the content of requests intended for downloading the corresponding files from the HTTP- and FTP-servers. Network packets containing these strings were transformed according to the scenarios below. To generate rules for the Bro system, we have used the python script snort2bro.

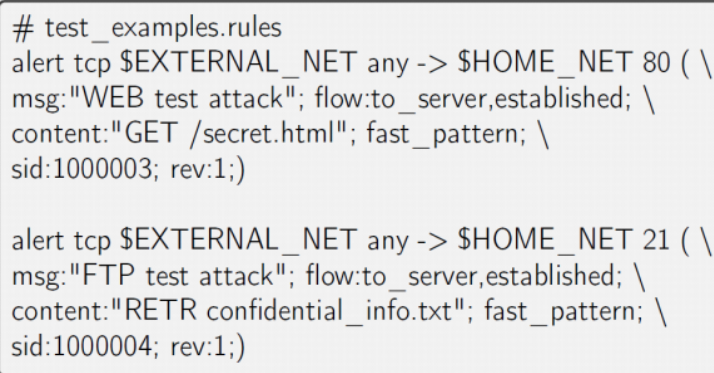

\section{Figure 5: Test examples of Snort signature rules.}

For conducting the network attacks, we have prepared 25 scenarios, which implement various weaknesses or limitations which are not strictly specified by the protocol. The choice of such attack scenarios is due to their simple implementation and the best coverage of several, namely four, levels of the OSI model. The first scenario affects the channel layer, the second to tenth scenarios implement the non-explicitly regulated features of the network IP-protocol, the scenarios with the numbers 11-24 describe some possible gaps in constructing the ADS network stack at the transport level, the 25 -th scenario describes a possible attack at the application level.

Below is a summary of the attack scenarios under consideration:

1. Replace the sender's hardware address for one of the packets of the established TCP/IP-session (spoofing the sender's MAC-address). 
2. Construct and send one of the TCP / IP session packets with the incorrectly calculated IP header checksum.

3. Send one of the TCP/IP-session packets in several IPfragments in descending order of IP-offset field values.

4. Split one of the TCP/IP-session packets at the IP-protocol level into several packets with partially overlapping data (it has been experimentally established that for Linux in the case of partially overlapping data, preference is given to packets that arrived earlier, or to be more precise, possessed a lower value of the IP-offset field inside the original datagram; Windows does not at all handle partially overlapping packets).

5. Split one of the TCP/IP-session packets at the IP-protocol level into three fragments: the first fragment contains the wrong checksum of the IP-header and randomly generated data, the second fragment contains the last half of the signature data, the third fragment completely overlaps the first fragment, but this time contains the correct checksum of the IP-header and the first half of the signature data (OS stack ignores the first fragment, replaces it with the third, and appends it with the second).

6. Split one of the TCP/IP-session packets at the IP-protocol level into three fragments: the first fragment contains randomly generated data, the second fragment has the same parameters as the first fragment, but with the required first half of the signature data (thereby the segment completely overlaps them), the third fragment contains the second half of the signature data (it has been experimentally established that for Linux in the case of completely overlapping IP-fragments, a higher priority is set for packets received later; for Windows - vice versa).

7. Send one of the IP-fragments with a specified time delay (in the experiments we have used the timeout value, not less than 30 seconds and 59 seconds, used by default in Linux and Windows respectively before sending an ICMP-packet with type 11 "Time-to-live exceeded" and code 1 "Fragment reassembly time exceeded").

8. Send an IP-datagram with empty flags.

9. Send an IP-datagram with an IP-header length field value of less than 20 bytes.

10. Send an IP-datagram with a maximum value of the length field of the IP-packet, equal to 65535 bytes.

11. Construct and send one of the TCP/IP-session packets with an incorrectly calculated TCP-checksum.

12. Send multiple TCP-packets in the reverse order of sequence numbers.

13. Send TCP-packets with partial layering on previously sent data inside TCP-packets (it has been experimentally established that for Linux and Windows, a higher priority is assigned to packets with the lowest sequence number),

14. Split one TCP/IP-session packet at the TCP-protocol level into three segments: the first segment contains an incorrect checksum of the TCP-pseudo-header and randomly generated data, the second segment contains the last half of the signature data, the third segment completely overlaps the first segment, but this time contains the correct checksum of the TCP-pseudo-header and the first half of the signature data (OS stack ignores the first segment, replaces it with the third, and appends it with the second).

15. Split one TCP/IP-session packet at the TCP-protocol level into three segments: the first segment contains randomly generated data and empty flags (receiving the packet without an established ACK-flag within the already established TCP-session is ignored by the OS), the second segment contains the second half signature data, the third segment has the same parameters as the first segment, but with the desired first half of the signature data (thereby the segment completely overrides it) and with the ACK-flag set (OS successfully confirms the reception of the third segment and appends it with the second).

16. Send one of the TCP-segments with the specified time delay (in the experiments we have used a timeout value of at least 20 seconds, used in apache 2 for Linux by default before sending the FIN-packets for terminating the connection; in the case of Windows, the same timeout value did not break connections to the nginx server).

17. Send payload data in the first TCP-session packet with the SYN-flag set (socket option MSG_FASTOPEN, RFC 7413 [19]).

18. Send payload data in the third packet within the "triple handshake" of the TCP-state machine with the ACK-flag set.

19. Send payload data in the FIN-packet of a TCP-session.

20. Send payload data in the RST-packet of a TCP-session.

21. Send payload data in the TCP-packets with unset TCPflags.

22. Send payload data in the TCP-packet with set TCP-flags URG|FIN|PSH|ACK ("XMAS-tree").

23. Send a TCP-packet with a TCP-header length value less than 20 bytes.

24. Send data in a TCP-packet with a sequence number less than the initial sequence number (ISN) received from the initiator of the session (fall over outside the session).

25. Insert the control character sequences before loading commands in HTTP and FTP text application protocols (in experiments we have used the sequence of hexadecimal character codes 0x0D 0x0A 0x0D 0x0A, i.e. " $|r| n \backslash r \backslash n ")$

Attacks related to manipulating the value of the IP-field of the TTL are not considered because ADS and the OS are on the same host. For a more detailed description of other different techniques for deception of network ADSs the reader can refer to the classic paper written by Ptacek and Newsham [13]. Table 3 presents the results of testing the ADSs, which were installed on the OSs Debian Jessie (8.4) and Windows 7.

All three ADSs were launched with the default settings, which already included options for processing and assembling the fragmented IP-packets.

The symbol "+" indicates the scenarios that cause triggering the ADS signature rule or result in a response packet from the server. The symbol "-" means that the rule ADS or server does not respond to the incoming packet. The scenarios are marked by the symbol "*" if packet analyzer of ADS functions correctly by warns about anomalous packets. Moreover we have labeled by the symbol "?" those scenarios which are uncharacteristic for the service (e.g. for protocol FTP it is not possible to load the file without performing the preliminary authorization procedure on the server, therefore the scenarios 17 and 18 are skipped for FTP).

For ease of reading some cells are painted by a light-gray color which means that ADS has a false positive alarm. The darkgray color of cell corresponds to the case that the ADS skips the network attack. Based on the results of experiments shown in Table 3, we can say that all three ADSs successfully cope with defragmentation and reassembly of IP-fragments and TCPsegments, respectively (scenarios 3, 4, 12, 13).

Two ADSs, Snort and Bro, have two similar drawbacks when processing packets which have arrived after the time allocated for their reassembling (scenarios 7 and 16). Suricata, in turn, correctly tracks ICMP- and TCP-packets that signal the shutdown of the 
network stream transmission channel from the server side (receiving of packets and acknowledgment of their receipt is allowed by the server). In Snort's functioning we have identified five false positives that correspond to scenarios 7, 16, 20, 21 and 22. With the help of such attacks an attacker can mislead deliberately the security analysts, diverting them to a vain search for an absent anomaly. Such attacks are a special case of insertion attacks: ADS reacts to packets of this type, but the host OS ignores their processing. Besides Snort does not detect 2 attack scenarios (6-th and 16-th). In the case of FTP for scenario 6 the ADS Snort may be deceived with the help of setting the large time-out. We have experimentally established that Snort does not detect the attack scenario 6 because this ADS uses the principle of IP-packet defragmenting which is characteristic for Windows.
Although the network stacks of Linux and Windows use an absolutely opposite mechanism of defragmentation in case of conflict which is described by the scenario 6 . To eliminate this disadvantage, it is necessary to specify explicitly the parameter "policy" for the preprocessor frag3_engine. For the ADS Suricata in the case of this scenario the opposite picture is observed.

The ADS Suricata is characterized by the greatest informativeness in the output of log-messages about detected network anomalies, in particular, it notifies about packets with a bad checksum, with overlapping parts of IP- and TCP- packets, with an invalid header length. Only two serious shortcomings were found in the functioning of this ADS - it does not detect the attack scenarios 5 and 24. The scenarios 11, 21 and 22 make Suricata to generate the false positive alarms.

Table 3: Test results for ADSs launched on Debian Jessie (8.4) and Windows 7

\begin{tabular}{|c|c|c|c|c|c|c|c|c|c|c|c|c|c|c|}
\hline \multirow{3}{*}{$\begin{array}{c}\text { Attack } \\
\text { scenario }\end{array}$} & \multicolumn{8}{|c|}{ Debian Jessie (8.4) } & \multicolumn{6}{|c|}{ Windows 7} \\
\hline & \multicolumn{2}{|c|}{$\begin{array}{c}\text { Snort } \\
2.9 .8 .2\end{array}$} & \multicolumn{2}{|c|}{$\begin{array}{c}\text { Suricata } \\
3.0 .1\end{array}$} & \multicolumn{2}{|c|}{$\begin{array}{c}\text { Bro } \\
2.4 .1\end{array}$} & \multicolumn{2}{|c|}{$\begin{array}{c}\text { Linux } \\
3.16 .0-4\end{array}$} & \multicolumn{2}{|c|}{$\begin{array}{c}\text { Snort } \\
2.9 .8 .2\end{array}$} & \multicolumn{2}{|c|}{$\begin{array}{c}\text { Suricata } \\
3.0 .1\end{array}$} & \multicolumn{2}{|c|}{$\begin{array}{c}\text { Windows } \\
7\end{array}$} \\
\hline & 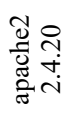 & 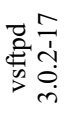 & 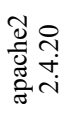 & 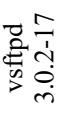 & 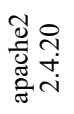 & 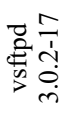 & 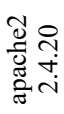 & 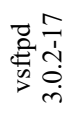 & 希号 & 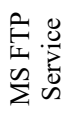 & 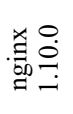 & 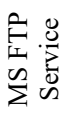 & 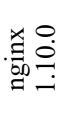 & 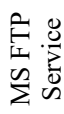 \\
\hline 1 & + & + & + & + & + & + & + & + & + & + & + & + & + & + \\
\hline 2 & - & - & - & - & - & - & - & - & - & - & * & $*$ & - & - \\
\hline 3 & + & + & + & + & + & + & + & + & + & + & + & + & + & + \\
\hline 4 & + & + & + & + & + & + & + & + & + & + & * & $*$ & - & - \\
\hline 5 & + & + & - & - & + & + & + & + & + & + & - & $\overline{-}$ & + & + \\
\hline 6 & - & - & + & + & $\overline{-}$ & $\overline{-}$ & + & + & + & + & $\overline{-}$ & - & + & + \\
\hline 7 & + & + & - & - & + & + & - & - & + & + & - & - & - & - \\
\hline 8 & + & + & + & + & + & + & + & + & + & + & + & + & + & + \\
\hline 9 & - & - & - & - & - & - & - & - & - & - & - & - & - & - \\
\hline 10 & - & - & - & - & - & - & - & - & - & - & - & - & - & - \\
\hline 11 & - & - & $*$ & $*$ & - & - & - & - & - & - & $*$ & $*$ & - & - \\
\hline 12 & + & + & + & + & + & + & + & + & - & $*$ & - & - & + & + \\
\hline 13 & + & + & + & + & + & + & + & + & + & + & - & - & + & + \\
\hline 14 & + & + & + & + & + & + & + & + & $\overline{-}$ & $*$ & $\overline{-}$ & $\overline{-}$ & + & + \\
\hline 15 & + & + & + & + & $\overline{-}$ & $\overline{-}$ & + & + & $\overline{-}$ & $*$ & $\overline{-}$ & $\overline{-}$ & + & + \\
\hline 16 & + & $\overline{-}$ & - & + & + & + & - & + & $\overline{-}$ & $*$ & $\overline{-}$ & $\overline{-}$ & + & + \\
\hline 17 & + & $?$ & + & $?$ & $\overline{-}$ & $?$ & + & $?$ & + & $?$ & + & $?$ & + & $?$ \\
\hline 18 & + & $?$ & + & $?$ & + & $?$ & + & $?$ & + & $?$ & + & $?$ & + & $?$ \\
\hline 19 & + & + & + & + & + & + & + & + & + & + & + & + & + & + \\
\hline 20 & + & + & - & - & - & - & - & - & + & + & $*$ & $*$ & - & - \\
\hline 21 & + & + & + & + & + & + & - & - & $*$ & $*$ & + & + & - & - \\
\hline 22 & + & + & + & + & + & + & - & + & + & + & + & + & + & + \\
\hline 23 & - & - & - & - & - & - & - & - & - & - & - & - & - & - \\
\hline 24 & + & + & - & - & - & - & + & + & + & + & + & + & + & + \\
\hline 25 & + & + & + & + & + & + & + & + & + & + & + & + & + & + \\
\hline
\end{tabular}


During the experiments it was found that Bro does not detect the attacks which are set by four scenarios $6,15,17,24$. The most significant weakness in the implementation of the Bro network analyzer is that it does not handle data transmitted in the first packet of TCP-session according to the scenario 17. Even after removing the keyword "established" in the corresponding signature, Bro still ignored such packages. Note that for Snort, detection of such evasion was decided by disabling this option. Therefore, an attack with concealment is possible here: ADS does not see an anomalous packet, and the target host, in turn, perceives it. This creates a secret passage which allows an attacker to bypass quietly the ADS and, without punishable consequences for him/her, to inject any malicious code into the attacked server. Also for Bro it was found that it mistakenly counts four scenarios with the numbers 7, 16, 21, 22 as attacks.

For the protected host Windows 7, the following results were obtained during the experiments. Snort skips four attack scenarios with the numbers $12,14,15,16$ and has three false positives caused by scenarios $4,7,20$. Suricata, in turn, skips seven attack scenarios with the numbers $5,6,12,13,14,15,16$ and assigns to attacking actions one false triggering, caused by the scenario 21 .

The recommendations for preventing such low-level network attacks are the following: (1) ignore fragmented IP-packets using a firewall: iptables -A INPUT "4\&0x3FFF=1:0x3FFF" -j DROP; (2) set the null timeout for assembling the fragmented packets together: echo $0>/$ proc/sys/net/ipv4/ipfrag_time; (3) replace the network ADSs by host-based analogues (OSSEC [20], Samhain [21]); (4) configure the network ADSs with settings which are as close as possible to the settings of the protected OS network stack.

\section{DISCUSSION}

Some of the features associated with the assembly of network packets are primarily due to the fact that the RFC 791 [22], RFC 1858 [23], RFC 3128 [24] do not specify the strict rules that describe the process of unified handling of such conflicting packets, which leads to some differences in the software implementation of network stacks on different OSs. In particular, according to RFC 791 in the case of identical fragments or fragments that overlap each other starting from the left border (that is, they have the same value of the IP-offset field), the highest priority should be given to packets received later, which in accordance with scenario 6 is consistent with the behavior of the Linux network stack. However, as shown experimentally, the network stack of Windows 7 contradicts this requirement and acts directly opposite. At the same time, for scenario 4 in the RFC, there are no explicit instructions for assembling packets that overlap partially, but they have a different IP-offset value.

Therefore, there is some arbitrariness in choosing which packet has to possess the highest priority. To implement correctly the procedure for removing expired IP-fragments, the ADS should either monitor the corresponding ICMP-packets that signal the expiration of the timeout allocated for the IP-packet assembly, or possess the same time-out value as the OS stack, e.g. obtained from the file /proc/sys/net/ipv4/ipfrag_time.

\section{CONCLUSIONS}

In this paper we have presented the model of checking the packet analyzer of network ADSs. On its basis we have developed a software tool for transparent proxying of TCP-sessions, designed for generating various low-level network attacks. In total, 25 attack scenarios were listed which cover four levels of the OSI model. Among such attacks we have considered formation of IPpackets, fragmented on the IP-level, sending of packets with delays, establishment of various TCP-flags, etc. The developed tool supports a client-server architecture and, unlike other wellknown analogues, aims to generate a wider variety of low-level network attacks, in particular evasion attacks and insertion attacks. We have proposed practical recommendations to prevent some of considered attacks. The results of the experiments showed that none of the considered network ADSs has the number of attack skips (false negative), equal to zero. In the future, it is planned to add functions of processing and forming of IP- and TCP-options into the asynchronous transparent proxy-server of TCP-sessions, to investigate other systems, including commercial ones, and also to perform a comprehensive testing of ADSs for their ability to detection of more complex, e.g. multi-step, attacks.

\section{ACKNOWLEDGMENTS}

This work was partially supported by grants of RFBR (projects No. 16-29-09482, 18-07-01488), by the budget (the project No. AAAA-A16-116033110102-5), and by Government of Russian Federation (Grant 08-08).

\section{REFERENCES}

[1] I. Kotenko. 2003. Active vulnerability assessment of computer networks by simulation of complex remote attacks. Proceedings of the International Conference on Computer Networks and Mobile Computing, 40-47.

[2] I. Kotenko, A. Chechulin. 2012. Common framework for attack modeling and security evaluation in SIEM systems. Proceedings of 2012 IEEE Int. Conf. on Green Computing and Communications, Conf. on Internet of Things, iThings 2012 and Conf. on Cyber, Physical and Social Computing, 94-101.

[3] D. Day, B. Burns. 2011. A performance analysis of snort and suricata network intrusion detection and prevention engines. Proceedings of the Fifth International Conference on Digital Society (ICDC), 187-192.

[4] C.A.P. Boyce, A.N. Zincir-Heywood. 2003. A Comparison of Four Intrusion Detection Systems for Secure E-Business. Proceedings of the International Conference on Electronic Commerce Research, 302-314.

[5] J.S. White, T. Fitzsimmons, J.N. Matthews. 2013. Quantitative analysis of intrusion detection systems: Snort and Suricata. Cyber Sensing, Vol. 8757.

[6] M. Ennert, E. Chovancová, Z. Dudláková. 2015. Testing of IDS model using several intrusion detection tools. Journal of Applied Mathematics and Computational Mechanics, Vol. 14, no. 1, 55-62.

[7] E. Albin. 2011. A comparative analysis of the snort and suricata intrusion detection systems. MA thesis, Naval Postgraduate School, Monterey CA.

[8] M.A.C. Moya. 2008. Analysis and evaluation of the Snort and Bro network intrusion detection systems. Bachelor's Thesis. Universidad Pontificia Comillas.

[9] J.T. Rødfoss. 2011. Comparison of open source network intrusion detection systems. University of Oslo, Department of Informatics.

[10] Snort. https://www.snort.org/.

[11] Suricata. https://suricata-ids.org/.

[12] Bro. https://www.bro.org/.

[13] T.H. Ptacek, T.N. Newsham. 1998. Insertion, evasion, and denial of service: Eluding network intrusion detection. SECURE NETWORKS INC CALGARY ALBERTA

[14] S. Northcutt, J. Novak. 2002. Network intrusion detection. Sams Publishing.

[15] SendIP. https://www-x.antd.nist.gov/ipv6/sendip.html.

[16] Hping. http://www.hping.org/.

[17] Fragrouter. https://tools.kali.org/information-gathering/fragrouter.

[18] Fragroute. https://www. monkey.org/ dugsong/fragroute/.

[19] Y. Cheng, J. Chu, S. Radhakrishnan, A. Jain. 2014. RFC 7413. TCP Fast Open. https://www.ietf.org/rfc/rfc7413.txt.

[20] OSSEC. https://www.ossec.net/.

[21] Samhain. https://la-samhna.de/samhain/.

[22] J. Postel. 1981. RFC 791. Internet protocol. https://www.ietf.org/rfc/ rfc791.txt.

[23] G. Ziemba, D. Reed, P. Traina. 1995. RFC 1858. Security Considerations for IP Fragment Filtering. https://www.ietf.org/rfc/rfc1858.txt.

[24] I. Miller. 2001. RFC 3128. Protection Against a Variant of the Tiny Fragment Attack. https://www.ietf.org/rfc/rfc3128.txt. 